\title{
L'utopie au XVIIIe siècle : côtoiements du politique, dynamiques de l'ailleurs, questionnements et dépassements des modèles utopiques classiques
}

\author{
Jean-Jacques Tatin-Gourier \\ Université de Tours
}

Le terme "ailleurs" est vague : les notions d'altérité, d'extériorité voire d'éloignement qu'il implique conviennent en un sens aisément à l'utopie et à ses spécificités génériques. De plus l'utopie à l'âge classique comme au siècle des Lumières constitue bien un "ailleurs" de la politique et de ses diverses problématiques (droit naturel, théories contractuelles, discours absolu et théorie du droit divin).

Mais comme dans tout exposé sur l'utopie, il importera ici tout d'abord de prendre ses distances à l'égard d'usages très extensifs et en fin de compte abusifs du terme utopie et de ses dérivés (utopique, utopiste). Le XVIIIe siècle préférait à ces qualifications polémiques et dégradatrices les termes "chimères" et "chimériques". La péjoration actuelle impliquée par le terme utopie et par ses dérivés est notamment héritée, on le sait, d'un certain marxisme qui a élaboré la grande ligne de partage entre "socialisme utopique" et "socialisme scientifique". Le "socialisme utopique" étant au mieux, dans le passé, la préfiguration du "vrai" socialisme, au pire, dans le présent, sa caricature et sa perversion.

Cette grille de lecture a été, et demeure parfois encore, projetée sur le champ littéraire et philosophique du XVIIIe siècle et du début du XIXe siècle (Barbéris 175). Quand l'on parcourt les commentaires et critiques suscités par les grandes figures des Lumières, un premier constat est flagrant : les qualifications d'utopique et d'utopiste sont essentiellement réservées à Jean-Jacques Rousseau penseur politique et pédagogue (le Contrat social et l'Émile sont avant tout visés). Ces qualifications sont par ailleurs souvent liées au procès de l'étrangeté" voire de la "folie" de Rousseau. Et l'on oppose volontiers au citoyen de Genève, le pragmatique Montesquieu, qui, bien loin d'"écarter les faits" (selon la formule inaugurale de Rousseau dans le Contrat social) s'attache, dans l'Esprit des lois, à démêler l'écheveau complexe des institutions existantes ou ayant existé à travers l'histoire. Le pragmatisme politique de Voltaire - par delà son attachement aux principes de la tolérance - ne fait lui aussi aucun doute : le propagandiste du modèle politique anglais dans les Lettres philosophiques n'est-il pas devenu le chantre des absolutismes éclairés européens?

Il me semble indispensable en préambule même de cet exposé de rejeter cette ligne de partage et de mettre les choses au clair quant aux rapports complexes et ambigus de Jean-Jacques Rousseau et de son œuvre politique (plus précisément du Contrat social, ouvrage de droit politique) avec la problématique utopique. J'évoquerai ensuite la nature disparate des textes dits "utopiques" et de leurs relations avec la thématique politique pour mettre en évidence un ailleurs de la politique contemporaine. L'uchronie et les séquences utopiques à l'oeuvre dans le roman seront finalement évoquées pour exposer la nature dynamique de la notion d'utopie au XVIIIe siècle ainsi que la crise qui en affecte sa problématique même. 


\section{Rousseau : la hantise de l'utopie, la mise en scène de ses limites et l'élaboration des concepts ouvrant la voie d'un ailleurs politique.}

Dans la société comme dans l'éducation, Rousseau élabore un contrat qui, par delà des situations historiques variables, est toujours susceptible d'entraîner les hommes vers un ailleurs de la politique de fait, à quelque étape du devenir historique que ce soit. Avec cette problématique du droit politique, nous sommes donc très éloignés des textes utopiques classiques qui nous donnent à lire une totalisation, une cité dans son achèvement et sa perfection. Totalité dans laquelle, on le sait, l'homme qui y trouve sa place, connaît à la fois la justice et le bonheur.

On considère souvent que Rousseau a plus précisément cédé à son goût pour l'utopie dans la Nouvelle Héloïse. Et l'on évoque généralement l'espace de Clarens dont Julie est la grande ordonnatrice. Or c'est précisément dans ce roman épistolaire au succès exceptionnel, au cœur même de la diégèse et de sa dynamique, que Rousseau questionne l'utopie et met en lumière ses limites. De Clarens au "pays des chimères" évoqué à la fin du roman (sans oublier "le ciel" et sa pureté auxquels donnerait accès l'œuvre de "Jean-Jacques" dans le Dialogue de Rousseau juge de Jean-Jacques 819, 829), il est évident que l'utopie hante la pensée de Rousseau. Bien des séquences des autres œuvres de Rousseau témoignent de cette hantise. Mais prenons garde : Clarens que l'on présente souvent comme utopie essaimée au sein du roman épistolaire est foncièrement ancrée dans le réel, participe pleinement de la réalité économique et sociale, de son ordre, de ses règles et de ses interdits. Et pour Saint-Preux, à Clarens, Julie épouse de M. de Wolmar est plus que jamais inaccessible : elle proclame de plus à l'heure de sa mort qu'il n'y a de beau que ce qui n'est pas.

L'utopie de Clarens n'est en fait qu'illusion précaire et donc condamnée parce que minée par une réalité modelée par le passé et toujours prête à ressurgir. Georges Benrekassa écrit dans l'article «Utopies des lumières » de l'Histoire littéraire de la France :

A la limite, on pourrait dire qu'il n'y a dans La Nouvelle Héloïse qu'un "lieu utopique" : c'est l'Élysée de Julie, objet de la lettre XI de la quatrième partie, lieu mystérieux, lieu de récréation strictement réservé à des élus : lieu où l'efflorescence a été organisée pour sembler libre, et où tout d'ailleurs a été calculé pour donner l'illusion du désert; lieu tout à fait hors du monde, d'où son nom et le seul où, de son propre aveu, Saint-Preux peut éprouver complètement la jouissance de la vertu, qui suppose la jouissance de soi. Du même coup, on voit bien que ce lieu utopique est vide et désigne sa vacuité aussi bien que les noms de lieux jadis forgés par Thomas More (t.6 141)

La dynamique même du roman épistolaire atteste que La Nouvelle Héloïse est démonstration du caractère précaire et illusoire de l'utopie et de la nécessité de son dépassement. Et c'est précisément là qu'interviennent, avec le Contrat social et l'Emile, dont la publication suit immédiatement celle de La Nouvelle Héloïse, les 
perspectives contractuelles du droit politique. Considérer la cité du Contrat social, promouvant et respectant ses fameux "principes" comme une utopie, c'est oublier

1. que Rousseau a écrit son ouvrage de droit politique au terme d'une longue réflexion sur l'histoire (c'est l'objet du Discours sur les sciences et les arts et du Discours sur l'origine de l'inégalité) et que le Contrat social énonce précisément les principes et concepts susceptibles de surmonter les malheurs et les injustices engendrées par cette histoire. Le Contrat social se propose de rectifier, de corriger les effets du faux contrat qui, dans le Discours sur l'origine de l'inégalité, a mis l'État au service du riche et consacré et renforcé des rapports de violence et d'oppression en quelque sorte légalisés par ce faux contrat.

2. C'est aussi oublier que le Contrat social est suivi d'applications très concrètes (Projet de constitution de la Corse, Considérations sur le gouvernement de Pologne). Ainsi on ne retrouve nullement dans l'œuvre politique de Rousseau l'isolisme et le fixisme qui caractérisent l'utopie. Au XVIIIe siècle, la pensée politique de Rousseau est sans doute la plus révélatrice, non seulement de la crise contemporaine de l'utopie, mais peutêtre plus encore de la dynamique de son dépassement.

\section{Un corpus de textes formellement disparates et généralement appréhendés aujourd'hui comme textes utopiques.}

Au delà des usages manifestement abusifs du terme "utopie"-"usages abusifs" qui conduisent à qualifier Rousseau qui fait œuvre de droit politique d'utopiste-, il importe de réfléchir à notre manière de constituer le corpus des œuvres du XVIIIe siècle que nous identifions comme utopies.

Un premier constat s'impose : il s'agit de textes formellement très disparates. Et force est de constater aussi qu'au fil des deux siècles qui nous séparent des Lumières, ce corpus a été véritablement à géométrie variable, notamment avec l'impact, à la fin du XIXe siècle, de la dichotomie du "socialisme utopique" et du "socialisme scientifique". Au XVIIIe siècle même, les ambiguïtés ne manquent pas. Certes le terme utopie renvoie d'abord au texte de Thomas More et au schéma narratif qu'il implique ${ }^{1}$, mais en même temps les définitions contemporaines tendent à embrasser un champ plus ample - et notamment un champ proprement politique - et à envisager des textes-sources plus anciens :

... on le dit quelquefois figurément du plan d'un gouvernement imaginaire à l'exemple de la République de Platon.

affirme l'édition de 1762 du Dictionnaire de l'Académie à la notice "utopie". De plus, à l'époque, certains textes que nous considérons de nos jours comme utopiques, se trouvent dispersés dans des catégories diverses : romans philosophiques, romans politiques, et plus tard, dans les volumes d'Économie

\footnotetext{
${ }^{1}$ Sur le mode narratif des textes utopiques, voir l'ouvrage de Jean-Michel Racault.
} 
politique et diplomatique (1788) de l'Encyclopédie méthodique, la catégorie d'“administration théorique". Il y a enfin le grand fourre-tout (36 volumes) élaboré par Charles Garnier, les Voyages imaginaires, songes, visions, et romans cabalistiques $^{2}$ où se trouvent étrangement réunis le Diable amoureux de Cazotte et la séquence des Troglodytes des Lettres persanes. Comme si la thématique de l'AILLEURS était le seul dénominateur commun de cet immense recueil.

\section{De l'interférence avec les discours ambiants à la mise en scène d'une altérité absolue, résolument extrapolitique.}

Il est un texte du midi du siècle (1755), texte souvent évoqué qui atteste que l'horizon de l'utopie demeure pour une part foncièrement politique : il s'agit du Code de la nature de Morelly, d'ailleurs longtemps attribué à Diderot et que Babeuf revendiquera encore à la fin du siècle comme inspirateur de sa conspiration des égaux. Mais il ne faut pas séparer ce texte abstrait, politique théorique du roman utopique du même Morelly, La Basiliade dont il est littéralement déduit. ${ }^{3}$ Dans un commentaire abstrait du roman, Morelly déduit de la législation de Zeinzenim exposée dans La Basiliade, "les règles d'un très sage gouvernement" qui rompt tout à la fois avec le despotisme des princes, la politique vulgaire et surtout la propriété dénoncée comme principe corrupteur de la société. (C'est d'ailleurs pour cette raison que Babeuf pourra, sous le Directoire, se réclamer de l'inspiration du Code de la nature de Morelly.)

Dans les utopies du XVIIIe siècle il est toutefois bien d'autres proximités, d'autres proximités avec les courants de pensée politiques contemporains :

- avec la politique fénelonienne telle qu'elle apparaît dans le Télémaque ;

- avec les stéréotypes (voire les clichés les plus éculés) du discours de l'absolutisme éclairé ou avec les discours marqués par le modèle anglais. Et l'on pense à la Relation du royaume de Félicie (1727) du marquis de Lassay, à l'Entretien d'un Européen avec un Insulaire du royaume de Dumocala (1752) de Stanislas Leszczynski. L'An 2440 de Louis Sébastien Mercier (1784), texte très novateur par sa dimension d'uchronie (nous en reparlerons), reconduit lui aussi les leitmotive de l'absolutisme éclairé ;

- il est aussi des utopies physiocratiques : marquées par le schéma narratif de Thomas More mais présentant en fait une gestion de l'île conforme aux principes de la politique de la physiocratie, comme cela est le cas de L'isle inconnue de Grivel (1783).

Mais au-delà de ce conformisme politique de nombre d'utopies, la tendance à la mise en scène d'une altérité radicale à tout système politique connu se manifeste clairement. Cette altérité peut tenir à une simple absence de la politique,

\footnotetext{
239 tomes publiés de 1787 à 1789 .

${ }^{3}$ Naufrage des isles flottantes, ou Basiliade du célèbre Pilpai, 1753.
} 
à un simple effacement des institutions. Dans le Télémaque de Fénelon (16941690), les habitants de la Bétique, fraternels et heureux parce qu'ayant accès au Bien, semblent n'avoir nul besoin d'institutions politiques.

Mais bien des textes utopiques nous apparaissent comme radicaux parce qu'ils conduisent leurs lecteurs dans des espaces difficilement identifiables, dans de véritables ailleurs de la politique. Dans l'Histoire des Ajaoiens (1767 dont Fontenelle est probablement l'auteur), il n'est pas de fondateur ou de législateur. La religion fauteuse de tyrannie est exclue et supplantée par le matérialisme athée : “... la nature (est) à la place de ce que nous nommons Dieu.” Dans l'Histoire de Calejava ou De l'isle des Hommes raisonnables avec le parallèle de leur Morale et du Christianisme (1700) de Claude Gilbert, la religion a été supplantée par l'exercice de la raison. Limitation du travail, répartition collective des produits : on a bien affaire ici à un ailleurs de la politique avec ces questions nouvelles, ces points de vue inédits; ces thématiques de rupture. Il n'est évidemment rien d'étonnant que ces textes utopiques aient relevé de la littérature clandestine ou que leur publication ait longtemps été différée.

Les pièces dites utopiques de Marivaux nous conduisent elles aussi vers cet ailleurs de la politique contemporaine : inversion du rapport maitre/esclave dans l'Île des esclaves (1725), renversement du rapport social dans l'Île de la raison ou les Petits Hommes (1727), inversion du rapport de domination des sexes dans $L a$ Nouvelle Colonie ou la Ligue des femmes (1729). D'autres questions et thématiques apparaissent encore dans des gammes de textes de formes extrêmement diverses. Certes la Découverte australe de Rétif de la Bretonne (1776), qui nous intéressera par sa conception progressive du devenir historique, présente bien des traits de l'utopie classique (en particulier par la clôture de son premier espace, le Mont inaccessible. Elle débouche toutefois sur le récit d'une colonisation qui permet la formation d'hybrides, le croisement des hommes et des animaux les plus variés.

On est proche ici d'une production longtemps ignorée, rejetée voire niée parce qu'intégralement dominée par l'imaginaire d'une altérité absolue. On va bien au-delà du classique voyage sur la lune de Cyrano. Des titres comme Relation d'un voyage du pôle arctique au pôle antarctique par le centre du monde (1721) sont d'ailleurs révélateurs et la thématique du monde souterrain ${ }^{4}$ semble avoir connu un grand succès avec des ouvrages comme :

- Voyage de Nicolas Klein dans le monde souterrain (1741),

- Lamekis de Charles Fieux de Mouhy (1735-1737),

- L'empire des Zahiris (1761) de Tiphaigne de la Roche,

- L'Isocameron de Casanova (1788) avec le monde intérieur des

Mégamicres,

- Les Confessions de Cagliostro (1787. Texte apocryphe.). Le pseudo

Cagliostro affirme que son épouse est née dans un espace éclairé à

l'électricité situé sous les pyramides d'Égypte.

\footnotetext{
${ }^{4}$ A noter sur ce point la thèse de doctorat de Mme Priscille Doucet, soutenue en 2006 à l'université de la Sorbonne, Le monde souterrain et ses origines dans la littérature française du XVIIIe siècle, dont nous avons pris connaissance après la soumission de ce texte.
} 
Dans Le voyageur philosophe dans un pays inconnu aux habitants de la terre (1761) de Listonai (pseudonyme de Daniel de Villeneuve), la cité de Sélénopolis, située sur la lune, comporte tout ce qui manque aux créatures terrestres : les monuments détruits de l'antiquité, les cartes géographiques encore manquantes, les inventions à attendre de l'avenir.

A ce point extrême de la dynamique de l'ailleurs, l'on comprend mieux, audelà des fantastiques réponses, ce qui se joue sous ce discours utopique qui a cessé d'être une simple dépendance directe du discours politique. Je citerai encore une fois Georges Benrekassa et son article « utopies des Lumières » :

Ce que l'utopie véhicule, ce sont finalement bien souvent des questions que, semble-t-il, le discours politique ou même le discours philosophique d'un temps, sont dans l'impossibilité de formuler ouvertement (127)

De la proximité des problématiques politiques contemporaines à son éloignement paroxystique, le corpus utopique du XVIIIe siècle donne ainsi à lire une indéniable et parfois surprenante dynamique de l'ailleurs.

\section{Rapprochement dans l'espace et passage à l'uchronie.}

Dans Lumières de l'utopie, Bronislaw Baczko a montré comment la cité utopique, dans le second versant du XVIIIe siècle tend à se faire plus proche en même temps que la conjonction avec la conception progressive du devenir historique s'affirme. Le texte le plus symptomatique de cette mutation est évidemment l'An 2440 (1784) de Louis Sébastien Mercier. Le voyageur visiteur est un Anglais et l'espace de ces déambulations n'est pas une île lointaine mais Paris qu'une révolution impulsée par un roi juste à métamorphosé : le Paris propre et prospère doté d'un sénat constitué d'hommes sages. Et nous sommes en l'année 2440 : la projection dans l'avenir s'est substituée à l'éloignement et à l'altérité de l'espace.

L'utopie peut être aussi tout simplement présente : c'est notamment le cas avec les communautés utopiques citadines ou paysannes que Rétif de la Bretonne évoque dans les Contemporaines (communauté parisienne et artisanale de la rue Saint Martin, communauté paysanne d'Oudun en Poitou, 1780). Les choses sont toutefois plus complexes avec la Découverte australe (1776) du même Rétif de la Bretonne. C'est en Dauphiné, sur le Mont inaccessible que Victorin transporte avec sa machine volante sa bien aimée Christine et les marginaux qu'il enlève dans les campagnes environnantes pour la servir. Mais au fil du temps, avec l'accroissement de la population, l'utopie est condamnée à se transporter au loin, dans l'île des antipodes que Victor baptise du nom de son épouse. Et l'île Christine elle-même, avec le temps, est contrainte d'étendre son espace et Victorin et ses fils créent le grand empire de Mégapatagonie qui se dotera d'une constitution. La thématique de l'altérité de l'espace et des êtres se développe ainsi dans un discours global dominé par l'idée de progrès (Loti). 


\section{Une implication de l'essaimage de noyaux utopiques dans le roman du XVIIIe siècle : la démonstration des limites de l'ailleurs et le cheminement vers un ailleurs de l'utopie.}

La présence de séquences utopiques dans les romans du siècle des Lumières a été souvent constatée. Les analyses de Bronislaw Baczko dans Lumières de l'utopie sont une fois encore très pertinentes sur ce point. Et outre Clarens dans la Nouvelle Héloïse, l'on cite généralement l'ample séquence des Troglodytes -plus exactement des bons Troglodytes puisque ces derniers se corrigent pour un temps d'une méchanceté foncière initiale- dans les Lettres persanes de Montesquieu, la réalisation de micro-sociétés (la Communauté d'Oudun et la Communauté de la rue Saint-Martin) dans les Contemporaines de Rétif de la Bretonne, les trois séquences utopiques dans Cleveland (1731-1739) de l'abbé Prévost (1'utopie huguenote et rocheloise de l'île de Sainte Hélène, l'utopie des Abaquis fondée et détruite par Cleveland lui-même et l'utopie des Nopandes). Enfin le plus célèbre noyau utopique dans l'un des romans les plus lus du siècle : l'Eldorado de Candide (1759).

C'est précisément en scrutant ces noyaux utopiques que l'on perçoit le plus clairement l'ampleur de la crise qui affecte la problématique même de l'utopie. En effet dans chacune de ces séquences, il y a démantèlement et littéralement dépassement d'une utopie qui finalement s'écroule ou s'efface pour faire place à un ailleurs de l'utopie. Et les raisons de cet anéantissement, de cet effacement et de ce dépassement sont toujours plus ou moins du même ordre : les contraintes de la société utopique sont toujours trop fortes, trop pesantes pour un sujet en quête d'un plaisir toujours plus fort, d'un bonheur toujours plus grand.

Dans la Nouvelle Héloïse, nous l'avons vu, Clarens disparaît avec la mort de Julie, mort qui est peut-être un suicide et qui constitue la réponse finale à d'indicibles aspirations que l'utopie domestique, bien ancrée dans la réalité familiale, économique et sociale ne pouvait pas satisfaire.

Candide, on le sait, s'éloigne de l'Eldorado avec Cacambo et ses gros moutons rouges chargés d'or et de pierreries pour retrouver le monde connu : l'Eldorado où l'or n'a aucune valeur, constitue une banalité du décor quotidien, ne peut satisfaire Candide que si ce dernier quitte cet espace utopique pour retrouver une monde où l'or est le signe le plus éminent de la richesse et du pouvoir.

Quant aux bons Troglodytes des Lettres persanes, la perfection de leur société n'aura été qu'un temps finalement assez bref de leur histoire mouvementée et décidément cyclique. Née de la lassitude et de l'épuisement de la guerre de tous contre tous -c'étaient alors les "méchants Troglodytes"-, leur société fraternelle, prospère mais aussi prolifique, les conduit à choisir un roi pour maintenir un ordre de plus en plus menacé. Et le plus âgé des Troglodytes pressenti pour devenir roi, déclare préférer mourir plutôt que d'envisager d'être l'artisan de ce retour à l'assujettissement et à l'oppression. Pour lui l'ailleurs de l'utopie est simplement la mort.

Mais c'est sans doute dans les trois séquences utopiques de Cleveland de l'abbé Prévost que le démantèlement et le dépassement de l'utopie par un sujet foncièrement libre et mobile apparaissent le plus clairement. Bridge, le frère lui aussi errant de Cleveland (Bridge et Cleveland fuient à travers le monde les 
persécutions de leur père Cromwell) débarque, avec quelques compagnons, dans une anse retirée de l'île de Sainte-Hélène. Il y est accueilli par une communauté huguenote formée par les réfugiés de La Rochelle exilés un siècle plus tôt. Contraints d'épouser les filles de la communauté que les autorités leur assignent, les voyageurs se rebellent et organisent une sédition qui sera fatale à la cité utopique. Quant à Cleveland qui s'improvise un temps législateur d'une tribu indienne, les Abaquis, il n'hésite pas à les abandonner à la férocité d'une tribu anthropophage - les Rouintons - et aux épidémies qui les déciment pour poursuivre sa route à travers les îles des Caraïbes. Mme Riding, compagne de Cleveland depuis l'enfance, errante elle aussi mais grande manipulatrice de toutes les intrigues, reçoit quant à elle l'hospitalité du prince des Nopandes, prince éclairé d'une petite cité fondée dans le passé par des voyageurs européens. Conseillère écoutée du prince, elle n'hésite pourtant pas à le trahir et à l'abandonner, laissant ce monde utopique dans un désarroi qui, de toute évidence, précipitera sa décadence. Cleveland, le roman de l'abbé Prévost, donne donc moins à lire trois états de l'utopie que trois formes de sa destruction par des êtres que meut un intérêt personnel, impitoyable, absolument cynique.

L'utopie au siècle des Lumières connaît ainsi une extension et une polyvalence sans précédent : essaimage dans divers genres littéraires - et essentiellement le roman -, projection vers l'avenir et passage à l'uchronie mais aussi élan vers l'ailleurs, réponses fantasmatiques à des questions souvent indicibles, informulables en un autre espace que l'espace utopique. Questions précisément informulables dans l'espace politique contemporain. C'est pourtant cet espace politique que l'utopie ne cesse de côtoyer, avec lequel elle ne cesse d'interférer. Et qu'elle rejoindra, avec des contenus très programmatiques et une écriture explicitement réglementaire au cours des années révolutionnaires. ${ }^{5}$

\section{Bibliographie}

Baczko, Bronislaw. Lumières de l'utopie. Paris, Payot, 1978.

Barbéris, Pierre. « Conscience moderne et société marchande, de Voltaire à

Voltaire en passant par Rousseau. René et la société marchande. » Histoire

\footnotetext{
${ }^{5}$ Je renvoie à l'article - dont le titre est d'ailleurs très révélateur - d'Anne-Rozenn Morel, « L'utopie au service de l'action politique » paru dans le volume Temps, durée dans la littérature des Lumières et ses marges. Voir également Bronislaw Baczko, Michel Porret et François Rosset, Dictionnaire critique de l'utopie au temps des lumières. Genève : Georg, 2016: 127-148.
} 
littéraire de la France, 1794-1830. Paris : Éditions sociales, 1976, t. 7 :

n.p.

Benrekassa, Georges. « Utopies des lumières. » Histoire littéraire de la France.

Paris : Éditions sociales, 1976, t. 6 : n.p.

Dictionnaire de l'Académie Française. Quatrième édition. Paris, 1762.

Fontenelle, de. La République des Philosophes ou Histoire des Ajaoiens. Première édition. s.n., 1768.

Gilbert, Claude. Histoire de Calejava ou De l'isle des Hommes raisonnables avec le parallèle de leur Morale et du Christianisme. Première édition. s.n., 1700.

Loty, Laurent. « La Découverte australe (1781): une utopie évolutionniste et eugéniste. » Etudes rétiviennes 4-5 (1986): 27-36.

Marivaux, Pierre Charlet de Chamberlain de. L'Île des esclaves. Paris : chez Noel Pissot, 1725.

---. L'Île de la raison ou les Petits Hommes. Paris : Prault père, 1727.

---. La Nouvelle Colonie ou la Ligue des femmes. Paris : s.n, 1729.

Montesquieu, Charles-Louis de Secondat. Lettres persanes. Paris : Alphonse Lemerre, 1721.

Morel, Anne-Rozenn. «L'utopie au service de l'action politique. » Temps, durée dans la littérature des Lumières et ses marges. Jean M. Goulemot (dir.). Université de Tours : Le Manuscrit, 2010 :127-148.

Morelly, Étienne-Gabriel. Code de la nature, ou Le véritable esprit de ses lois de tout temps négligé ou méconnu. Partout : chez le vrai sage, 1755.

Prévost, d'Exiles A.-F. Le philosophe anglois, ou histoire de Monsieur Cleveland, fils naturel de Cromwell. 4 tomes. Amsterdam et Leipzig : chez Arkstée \& Merkus, 1744

Racault, Jean-Michel. L'utopie narrative en France et en Angleterre (1675-1761). Oxford : SVEC, 1991.

Rétif de la Bretonne, Nicolas-Edme. Les contemporaines, ou Aventures des plus jolies femmes de l'âge présent : les contemporaines mêlées. Paris : J. Assezat, 1884.

---. La découverte australe par un homme volant, ou Le dédale français. Paris : s.n., 1781.

Rousseau, Jean-Jacques. « Rousseau juge de Jean-Jacques, Dialogues. » Oeuvres complètes. t. I. Pléiade, 1959.

Villeneuve, Daniel de. Le voyageur philosophe dans un pays inconnu aux habitants de la terre. Première édition. Amsterdam :s.n., 1761.

Voltaire, Candide ou l'optimisme. Paris : s.n., 1759. 